JUNE 1998, PAGES 201-219

\title{
LARGE-TIME BEHAVIOR OF SOLUTIONS TO THE EQUATIONS OF ONE-DIMENSIONAL NONLINEAR THERMOVISCOELASTICITY
}

\author{
BY \\ L. HSIAO AND T. LUO \\ Academia Sinica, Institute of Mathematics, Beijing 100080, China
}

1. Introduction. This paper is concerned with the investigation of large-time behavior of globally defined smooth solutions of the initial-boundary value problem for the system in one-dimensional nonlinear thermoviscoelasticity, namely,

$$
\left\{\begin{array}{l}
u_{t}-v_{x}=0 \\
v_{t}-\sigma_{x}=0 \\
{\left[e+\frac{1}{2} v^{2}\right]_{t}-[\sigma v]_{x}+q_{x}=0}
\end{array}\right.
$$

which is the referential (Lagrangian) description of the balance laws of mass, momentum and energy for one-dimensional materials with reference density $\rho_{0}=1$ and is supplemented with the second law of thermodynamics expressed through the Clausius Duhem inequality

$$
\eta_{t}+\left(\frac{q}{\theta}\right)_{x} \geq 0
$$

where $u, v, e, \sigma, \eta, \theta$, and $q$ denote specific volume (deformation gradient), velocity, internal energy, stress, specific entropy, temperature, and heat flux, respectively, while $e, \sigma, \eta$, and $q$ are given by so-called constitutive relations for the thermoviscoelastic materials to be considered. The quantities $u, \theta$, and $e$ may only take positive values.

We consider here a body with reference configuration the interval $[0,1]$ whose endpoints are stress-free and thermally insulated, that is,

$$
\left\{\begin{array}{l}
\sigma(0, t)=\sigma(1, t)=0, \\
q(0, t)=q(1, t)=0,
\end{array} \quad t \geq 0,\right.
$$

and we prescribe the initial values of $u, v$, and $\theta$ as follows:

$$
u(x, 0)=u_{0}(x), \quad v(x, 0)=v_{0}(x), \quad \theta(x, 0)=\theta_{0}(x), \quad 0 \leq x \leq 1 .
$$

For the material of ideal gas, in which the constitutive relations take the form

$$
e=c \theta, \quad \sigma=-R \frac{\theta}{u}+\mu \frac{v_{x}}{u}, \quad q=-K \frac{\theta_{x}}{u}
$$

Received February 1, 1995.

1991 Mathematics Subject Classification. Primary 73F15.

The first author was supported partially by the National Natural Science Foundation of China. 
where $c, R, \mu$, and $K$ are positive constants, it has been proved in [NA] that the solution $\left(u^{*}, v^{*}, \theta^{*}\right)$ to the problem (1.1), (1.3), and (1.4) satisfies

$$
u^{*}(x, t) \geq c^{*} \log (1+t), \quad c^{*}>0 .
$$

On the other hand, however, totally different phenomena may occur on large-time behavior of solutions for other kinds of constitutive relations to be considered. In the case of isothermal viscoelasticity (i.e., $\theta \equiv$ constant), the solution may approach a unique state exponentially fast as shown by Greenberg and MacCamy in [GM], or phase transition may take place as discovered by Andrews and Ball in [AB] with nonmonotone pressure, who prove that the large-time behavior of strain is described by a Yang measure whose support is confined in the set of zeroes of pressure. Our goal here is to extend the analysis to the nonisothermal case - thermoviscoelastic materials.

For simplicity, we consider in the present paper the kind of solid-like materials with the following constitutive relations:

$$
e=C_{V} \theta, \quad \sigma=-f(u) \theta+\hat{\mu}(u) v_{x}, \quad q=-k \frac{\theta_{x}}{u}
$$

where $C_{V}$ and $k$ are positive constants, and $f(u)$ is twice continuously differentiable for $u>0$ such that

$$
\begin{gathered}
f(u) \geq 0, \quad 0<u<\tilde{u} \\
f(u) \leq 0, \quad \tilde{U}<u<+\infty
\end{gathered}
$$

for some fixed $0<\tilde{u} \leq \tilde{U}<+\infty$, and the viscosity $\hat{\mu}(u) u$ is uniformly positive, that is,

$$
\hat{\mu}(u) u \geq \mu_{0}>0, \quad 0<u<+\infty .
$$

REMARK 1.0. It is known that for rubber a good model for pressure is of the form

$$
\hat{p}(u, \theta)=-\gamma \theta\left(u-\frac{1}{u^{2}}\right), \quad \gamma \text { is a positive constant }
$$

namely, $f(u)=-\gamma\left(u-\frac{1}{u^{2}}\right)$, which satisfies (1.7) with $\tilde{u}=\tilde{U}=1$.

We turn to assumptions on initial data now. Without loss of generality, by superimposing a trivial rigid motion, we normalize the initial velocity so that

$$
\int_{0}^{1} v_{0}(x) d x=0
$$

Furthermore, we assume that the initial data are compatible with the boundary conditions (1.3).

The global existence of (1.1), (1.3), and (1.4), under the assumptions of (1.6)-(1.9), can be established by the approach in $[\mathrm{DH}]$ and $[\mathrm{DA}]$ where the solid-like material with more general constitutive relations than (1.6) and (1.7) is concerned. Namely, assume $u_{0}(x), u_{0}^{\prime}(x), v_{0}(x), v_{0}^{\prime}(x), v_{0}^{\prime \prime}(x), \theta_{0}(x), \theta_{0}^{\prime}(x), \theta_{0}^{\prime \prime}(x)$ are in $C^{\alpha}[0,1]$ for some $0<\alpha<1$ and $u_{0}(x)>0, \theta_{0}(x)>0,0 \leq x \leq 1$; under the assumptions (1.6)-(1.9), there exists a unique solution $\{u(x, t), v(x, t), \theta(x, t)\}$ on $[0,1] \times[0, \infty)$ such that for every $T>0$, the functions $u, u_{x}, u_{t}, u_{x t}, v, v_{x}, v_{t}, v_{x x}, \theta, \theta_{x}, \theta_{t}, \theta_{x x}$ are all in $C^{\alpha, \frac{\alpha}{2}}\left(Q_{T}\right)$ and $u_{t t}, v_{x t}, \theta_{x t}$ are in $L^{2}\left(Q_{T}\right), Q_{T}=[0,1] \times[0, T]$. Moreover, $\theta(x, t)>0,0<\bar{u}<u(x, t)<\bar{U}$, for 
$0 \leq x \leq 1,0 \leq t<+\infty$, where $\bar{u}$ and $\bar{U}$ are positive constants depending only on the initial data but not on $T$, and $0<\bar{u} \leq \tilde{u} \leq \tilde{U} \leq \bar{U}$.

The following results on large-time behavior of solutions have been established in the present paper.

Theorem 1.1. Assume that (1.6)-(1.9) are satisfied. Let $\{u(x, t), v(x, t), \theta(x, t)\},(x, t) \in$ $[0,1] \times[0, \infty)$, be the globally defined smooth solution of the problem (1.1)-(1.4). Then I.

$$
\begin{aligned}
& \|\hat{p}(u, \theta)(\cdot, t)\|_{L^{1}[0,1]}=\|f(u) \theta(\cdot, t)\|_{L^{1}[0,1]} \rightarrow 0 \text { as } t \rightarrow+\infty \\
& \|f(u)(\cdot, t)\|_{L^{2}[0,1]} \rightarrow 0 \text { as } t \rightarrow+\infty \\
& \|v(\cdot, t)\|_{L^{2}[0,1]} \rightarrow 0 \text { as } t \rightarrow+\infty
\end{aligned}
$$

and

$$
\bar{\theta}(t) \stackrel{\text { def }}{=} \int_{0}^{1} \theta(x, t) d x \rightarrow \frac{E_{1}}{C_{V}} \quad \text { as } t \rightarrow+\infty
$$

where $E_{1}=\int_{0}^{1}\left[C_{V} \theta_{0}+\frac{1}{2} v_{0}^{2}\right]_{(x)} d x$.

II. There exists a family of probability measure $\left\{\nu_{x}\right\}_{x \in[0,1]}$ on $\mathbb{R}$ (depending measurably on $x)$ with supp $\nu_{x} \subset K=\{z: f(z)=0\}$ such that if $\Phi \in C(\mathbb{R})$ and

$$
g_{\Phi}(x) \stackrel{\text { def }}{=}\left\langle\nu_{x}, \Phi\right\rangle \text { a.e. }
$$

then $\Phi(u(\cdot, t)) \stackrel{*}{\rightarrow} g_{\Phi}(\cdot)$ in $L^{\infty}[0,1]$ as $t \rightarrow+\infty$.

REMARK 1.2. Theorem 1.1 extends the phase transition results in $[A B]$ to nonisothermal cases.

Corollary 1.3. Suppose the equation $f(z)=0$ possesses only one root $z=z_{1}$. Then

$$
u(\cdot, t) \rightarrow z_{1} \quad \text { strongly in } L^{q}(0,1) \text { as } t \rightarrow \infty
$$

for all $q, 1 \leq q<+\infty$, provided the conditions (1.6)-(1.9) hold.

Corollary 1.4. Suppose the equation $f(z)=0$ has exactly $m$ roots, $z_{1}, z_{2}, \ldots, z_{m}$, $m>1$. Then there exist nonnegative functions $\mu_{i} \in L^{\infty}[0,1], 1 \leq i \leq m$, such that

$$
\Phi(u(\cdot, t)) \stackrel{*}{\rightarrow} \sum_{i=1}^{m} \Phi\left(z_{i}\right) \mu_{i}(\cdot) \quad \text { in } L^{\infty}[0,1], \quad \text { as } t \rightarrow+\infty,
$$

for any $\Phi \in C(\mathbb{R})$.

Furthermore, $\sum_{i=1}^{m} \mu_{i}(x)=1$, a.e.

If $f(u)$ is strictly monotone decreasing, namely,

$$
f^{\prime}(u)<0 \text { for } u \in[\bar{u}, \bar{U}] \text {, }
$$

it follows from $(1.7)$ that there exists a unique $\hat{u} \in[\tilde{u}, \widetilde{U}]$ such that $f(\hat{u})=0$. We have further results then in the next theorem. 
ThEOREM 1.5. Assume that (1.6)-(1.10) hold. Then there are positive constants $\beta, \widehat{T}$, and $A$, independent of $t$, such that

$$
\|u(\cdot, t)-\hat{u}\|_{H^{1}(0,1)}+\|v(\cdot, t)\|_{H^{1}(0,1)}+\left\|\theta(\cdot, t)-\frac{E_{1}}{C_{V}}\right\|_{H^{1}(0,1)} \leq A e^{-\beta t} \quad \text { for } t \geq \widehat{T} .
$$

Theorem 1.5 generalizes the results obtained in [GM] which discusses the case of isothermal viscoelasticity.

Section 2 and Section 3 are devoted to proving Theorem 1.1 and Theorem 1.5, respectively.

2. The proof of Theorem 1.1. From now on, $\{u(x, t), v(x, t), \theta(x, t)\}$ will denote the solution described in the global existence theorem.

It is known from [DA] that

$$
0<\bar{u} \leq u(x, t) \leq \bar{U}, \quad \theta(x, t)>0, \quad x \in[0,1], \quad t \in[0,+\infty)
$$

where $\bar{u}$ and $\bar{U}$ are positive constants, independent of $t$, such that $0<\bar{u} \leq \tilde{u}<\widetilde{U} \leq \bar{U}$. (2.1) and (1.8) yield

$$
0<\mu_{1} \leq \hat{\mu}(u(x, t)) \leq \mu_{2}, \quad x \in[0,1], \quad t \in[0,+\infty)
$$

where $\mu_{1}$ and $\mu_{2}$ are positive constants, independent of $t$.

In the sequel, $\Lambda$ will denote a generic constant, independent of $t$.

Integrating $(1.1)$ over $[0,1] \times[0, t]$ and using the boundary condition (1.3) we obtain the conservation laws of total momentum and energy:

$$
\begin{gathered}
\int_{0}^{1} v(x, t) d x=\int_{0}^{1} v_{0}(x) d x=0, \quad 0 \leq t<+\infty \\
\int_{0}^{1}\left[C_{V} \theta+\frac{1}{2} v^{2}\right](x, t) d x=\int_{0}^{1}\left[C_{V} \theta_{0}+\frac{1}{2} v_{0}^{2}\right](x) d x=E_{1} .
\end{gathered}
$$

LEMMA 2.1.

$$
\int_{0}^{t} \int_{0}^{1}\left[\frac{\mu_{1} v_{x}^{2}}{\theta}+\frac{k \theta_{x}^{2}}{\theta^{2}}\right](x, \tau) d x d \tau \leq \Lambda, \quad t \in[0,+\infty) .
$$

Proof. Substituting $\sigma$ from (1.6), we may write $(1.1)_{2}$ in the form

$$
v_{t}+[f(u) \theta]_{x}=\left[\hat{\mu}(u) v_{x}\right]_{x}
$$

while combining $(1.1)_{3}$ with $(1.1)_{2}$ and using (1.6) we obtain

$$
C_{V} \theta_{t}+f(u) \theta v_{x}-\hat{\mu}(u) v_{x}^{2}-k \theta_{x x}=0 .
$$

Multiplying (2.7) by $\theta^{-1}$ and integrating over $[0,1] \times[0, t]$, with the help of $(1.3)$ and $(1.1)_{1}$, one obtains

$$
\begin{aligned}
& \int_{0}^{t} \int_{0}^{1}\left[\frac{\hat{\mu}(u) v_{x}^{2}}{\theta}+\frac{k \theta_{x}^{2}}{\theta^{2}}\right](x, \tau) d x d \tau \\
& \quad=C_{V}\left[\int_{0}^{1} \log \theta(x, t) d x-\int_{0}^{1} \log \theta(x, 0) d x\right]+\int_{0}^{1} G(u)(x, t) d x-\int_{0}^{1} G(u)(x, 0) d x
\end{aligned}
$$


where $G(u)=\int_{\bar{u}}^{u} f(\xi) d \xi$. This, with (2.1), (2.2), and the inequality $\log \theta \leq \theta-1$, for $\theta>0$, implies (2.5).

Due to (2.3) and the mean value theorem, there exists a $y(t) \in[0,1]$ for every $t \geq 0$ such that

$$
v(y(t), t)=0
$$

Thus

$$
|v(x, t)|=\left|\int_{y(t)}^{x} v_{x}(\xi, t) d \xi\right| \leq\left[\int_{0}^{1} \theta(x, t) d x\right]^{1 / 2}\left[\int_{0}^{1} \frac{v_{x}^{2}}{\theta}(x, t) d x\right]^{1 / 2},
$$

which, combined with $(2.4)$ and $(2.5)$, yields

$$
\int_{0}^{t} \max _{[0,1]} v^{2}(\cdot, \tau) d \tau \leq \Lambda, \quad t \in[0,+\infty)
$$

LEMMA 2.2.

$$
\int_{0}^{1}\left(v^{4}+\theta^{2}\right)(x, t) d x+\int_{0}^{t} \int_{0}^{1}\left[\theta_{x}^{2}+v^{2} v_{x}^{2}\right] d x d \tau \leq \Lambda, \quad t \in[0,+\infty) .
$$

Proof. Multiply $(1.1)_{3}$ with $\left(C_{V} \theta+\frac{v^{2}}{2}\right)$ and integrate over $[0,1] \times[0, t]$. With the help of $(1.3),(1.6),(2.1),(2.2)$, and Young's inequality, we arrive at

$$
\begin{gathered}
\frac{1}{2} \int_{0}^{1}\left[C_{V} \theta+\frac{v^{2}}{2}\right]^{2}(x, t) d x+\mu_{1} \int_{0}^{t} \int_{0}^{1} v^{2} v_{x}^{2}(x, \tau) d x d \tau+\frac{C_{V} k}{2} \int_{0}^{t} \int_{0}^{1} \theta_{x}^{2}(x, \tau) d x d \tau \\
\leq \Lambda+\Lambda \int_{0}^{t} \max _{[0,1]} v^{2}(\cdot, \tau) \int_{0}^{1} \theta^{2}(x, \tau) d x d \tau+\Lambda \int_{0}^{t} \int_{0}^{1} v^{2} v_{x}^{2}(x, \tau) d x d \tau
\end{gathered}
$$

To estimate the term $\int_{0}^{t} \int_{0}^{1} v^{2} v_{x}^{2} d x d \tau$, we multiply $(1.1)_{2}$ by $v^{3}$, integrate the resulting equation over $[0,1] \times[0, t]$, and use the boundary conditions $(1.3),(2.1),(2.2)$ and the Cauchy inequality. It then follows that

$$
\begin{gathered}
\frac{1}{4} \int_{0}^{1} v^{4}(x, t) d x+2 \mu_{1} \int_{0}^{t} \int_{0}^{1} v^{2} v_{x}^{2}(x, \tau) d x d \tau \\
\leq \Lambda+\Lambda \int_{0}^{t} \max _{[0,1]} v^{2}(\cdot, \tau) \int_{0}^{1} \theta^{2}(x, \tau) d x d \tau .
\end{gathered}
$$

By using the Cauchy inequality with the term $\left(C_{V} \theta+\frac{v^{2}}{2}\right)^{2}$ in $(2.12)$, we obtain

$$
\begin{gathered}
\int_{0}^{1} \theta^{2}(x, t) d x+\int_{0}^{t} \int_{0}^{1} v^{2} v_{x}^{2}(x, \tau) d x d \tau+\int_{0}^{t} \int_{0}^{1} \theta_{x}^{2}(x, \tau) d x d \tau \\
\leq \Lambda+\Lambda \int_{0}^{t} \int_{0}^{1} v^{2} v_{x}^{2}(x, \tau) d x d \tau+\Lambda \int_{0}^{t} \max _{[0,1]} v^{2}(\cdot, \tau) \int_{0}^{1} \theta^{2}(\cdot, \tau) d x d \tau \\
+\Lambda \int_{0}^{1} v^{4}(x, t) d x
\end{gathered}
$$


Multiplying (2.13) with a suitably large positive constant, and combining with (2.14), we get

$$
\begin{array}{r}
\int_{0}^{1} \theta^{2}(x, t) d x+\int_{0}^{1} v^{4}(x, t) d x+\int_{0}^{t} \int_{0}^{1} v^{2} v_{x}^{2}(x, \tau) d x d \tau+\int_{0}^{t} \int_{0}^{1} \theta_{x}^{2}(x, \tau) d x d \tau \\
\leq \Lambda+\Lambda \int_{0}^{t} \max _{[0,1]} v^{2}(\cdot, \tau) \int_{0}^{1} \theta^{2}(x, \tau) d x d \tau
\end{array}
$$

Applying Gronwall's inequality to (2.15) and using (2.10), one arrives at (2.11).

LEMMA 2.3.

$$
\int_{0}^{t} \int_{0}^{1} v_{x}^{2}(x, \tau) d x d \tau \leq \Lambda
$$

Proof. Multiplying $(1.1)_{2}$ by $v$ and integrating over $[0,1] \times[0, t]$, it follows, with the help of (1.3), (2.1), and (2.2), that

$$
\frac{1}{2} \int_{0}^{1} v^{2}(x, t) d x+\mu_{1} \int_{0}^{t} \int_{0}^{1} v_{x}^{2}(x, \tau) d x d \tau \leq \Lambda+\int_{0}^{t} \int_{0}^{1} f(u) \theta v_{x} d x d \tau
$$

Due to $(1.1)_{1}$ and $(2.1)$,

$$
\begin{aligned}
\int_{0}^{t} \int_{0}^{1} f(u) \theta v_{x} d x d \tau & \\
= & \int_{0}^{t} \int_{0}^{1} f(u)(\theta-\bar{\theta}) v_{x}(x, \tau) d x d \tau+\int_{0}^{t} \int_{0}^{1} f(u) \bar{\theta} v_{x}(x, \tau) d x d \tau \\
\leq & \frac{\mu_{1}}{4} \int_{0}^{t} \int_{0}^{1} v_{x}^{2}(x, \tau) d x d \tau+\Lambda \int_{0}^{t} \int_{0}^{1}(\theta-\bar{\theta})^{2}(x, \tau) d x d \tau \\
& +\int_{0}^{t} \int_{0}^{1} f(u) \bar{\theta} u_{t}(x, \tau) d x d \tau
\end{aligned}
$$

where $\bar{\theta}(t)=\int_{0}^{1} \theta(x, t) d x, t \in[0,+\infty)$.

By the mean value theorem, there exists a $z(t) \in[0,1]$ such that $\bar{\theta}(t)=\theta(z(t), t)$. Thus

$$
|\theta-\bar{\theta}|(x, \tau)=\left|\int_{z(\tau)}^{x} \theta_{x}(\xi, \tau) d \xi\right| \leq\left[\int_{0}^{1} \theta_{x}^{2}(x, \tau) d x\right]^{1 / 2}, \quad \tau \in[0,+\infty) .
$$

Integrating $(1.1)_{3}$ over $[0,1]$ and using (1.3) and (1.6), we arrive at

$$
C_{V}\left(\int_{0}^{1} \theta(x, \tau) d x\right)_{t}=-\left(\int_{0}^{1} \frac{1}{2} v^{2}(x, \tau) d x\right)_{t}, \quad \tau \in[0,+\infty) .
$$

Namely,

$$
\bar{\theta}_{t}(\tau)=-\frac{1}{C_{V}}\left[\int_{0}^{1} \frac{1}{2} v^{2}(x, \tau) d x\right]_{t}, \quad \tau \in[0,+\infty)
$$


which, together with (2.4), implies

$$
\bar{\theta}(\tau)=\frac{1}{C_{V}}\left[E_{1}-\int_{0}^{1} \frac{1}{2} v^{2}(x, \tau) d x\right], \quad t \in[0,+\infty) .
$$

In view of $(1.1)_{1},(2.1),(2.4),(2.10),(2.21)$, and (2.22), it follows, upon integrating by parts and using the Cauchy inequality, that

$$
\begin{aligned}
& \int_{0}^{t} \int_{0}^{1}\left[f(u) \bar{\theta} u_{t}\right](x, \tau) d x d \tau \\
& \leq \Lambda-\int_{0}^{t} \int_{0}^{1}\left\{f^{\prime}(u) u u_{t}\left[\frac{1}{C_{V}}\left(E_{1}-\int_{0}^{1} \frac{1}{2} v^{2}(s, \tau) d s\right)\right]\right\}(x, \tau) d x d \tau \\
&+\int_{0}^{t} \int_{0}^{1}\left\{f(u) u\left[\frac{1}{C_{V}}\left(\int_{0}^{1} \frac{1}{2} v^{2}(x, \tau) d s\right)\right]_{\tau}\right\}(x, \tau) d x d \tau \\
&=-\frac{E_{1}}{C_{V}}\left[\int_{0}^{1} \lambda(u)(x, t) d x-\int_{0}^{1} \lambda(u)(x, 0) d x\right] \\
&+\int_{0}^{t} \int_{0}^{1}\left[f^{\prime}(u) u v_{x}\left(\int_{0}^{1} \frac{1}{2} v^{2}(s, \tau) d s\right)\right](x, \tau) d x d \tau \\
&+\frac{1}{C_{V}}\left\{\int_{0}^{1}\left[f(u) u\left(\int_{0}^{1} \frac{1}{2} v^{2}(s, t) d s\right)\right](x, t) d x\right. \\
&\left.-\int_{0}^{1}\left[f(u) u\left(\int_{0}^{1} \frac{1}{2} v^{2}(s, 0) d s\right)\right](x, 0) d x\right\} \\
&-\frac{1}{C_{V}} \int_{0}^{t} \int_{0}^{1}\left\{\left[f^{\prime}(u) u+f(u)\right] v_{x}\left(\int_{0}^{1} \frac{1}{2} v^{2}(s, \tau) d s\right)\right\}(x, \tau) d x d \tau \\
& \leq \Lambda+\frac{\mu_{1}}{4} \int_{0}^{t} \int_{0}^{1} v_{x}^{2}(x, \tau) d x d \tau+\Lambda \int_{0}^{t} \int_{0}^{1} v^{4}(x, \tau) d x d \tau+\frac{1}{4} \int_{0}^{1} v^{2}(x, t) d x \\
& \leq \Lambda+\frac{\mu_{1}}{4} \int_{0}^{t} \int_{0}^{1} v_{x}^{2}(x, \tau) d x d \tau+\frac{1}{4} \int_{0}^{1} v^{2}(x, \tau) d x
\end{aligned}
$$

where

$$
\lambda(u)(x, t)=\int_{\bar{u}}^{u(x, t)} f^{\prime}(\xi) \xi d \xi
$$

(2.16) then follows from $(2.15),(2.17)-(2.19)$, and (2.23).

LEMMA 2.4.

$$
\int_{0}^{t} \int_{0}^{1}[f(u) \theta]^{2}(x, \tau) d x d \tau \leq \Lambda
$$


Proof. By integrating $(1.1)_{2}$ over $[0, x]$ for any $x \in[0,1]$ and using the boundary condition (1.3), it follows that

$$
[f(u) \theta](x, t)=\left[\hat{\mu}(u) v_{x}\right](x, t)-\left(\int_{0}^{x} v(y, t) d y\right)_{t}, \quad x \in[0,1], t \in[0,+\infty)
$$

Multiplying $(2.25)$ by $f(u) \theta$ and integrating it over $[0,1] \times[0, t]$, we arrive at

$$
\begin{aligned}
\int_{0}^{t} \int_{0}^{1}[f(u) \theta]^{2}(x, \tau) d x d \tau \\
\quad=\int_{0}^{t} \int_{0}^{1}\left[\hat{\mu}(u) v_{x} f(u) \theta\right](x, \tau) d x d \tau \\
\quad-\int_{0}^{t} \int_{0}^{1}\left\{\left[\int_{0}^{x} v(y, \tau) d y\right]_{\tau} f(u)(\theta-\bar{\theta})\right\}(x, \tau) d x d \tau \\
\quad+\int_{0}^{t} \int_{0}^{1}\left\{\left[\int_{0}^{x} v(y, \tau) d y\right]_{\tau} f(u) \bar{\theta}\right\}(x, \tau) d x d \tau
\end{aligned}
$$

We estimate each term in (2.26) separately.

In view of (2.1), (2.16), and Cauchy's inequality,

$$
\begin{aligned}
& \left|\int_{0}^{t} \int_{0}^{1}\left[\hat{\mu}(u) v_{x} f(u) \theta\right](x, \tau) d x d \tau\right| \\
& \quad \leq \frac{1}{4} \int_{0}^{t} \int_{0}^{1}[f(u) \theta]^{2}(x, \tau) d x d \tau+\Lambda, \quad t \in[0,+\infty)
\end{aligned}
$$

By (2.1), (2.11), (2.16), (2.19), (2.25), and the Cauchy inequality,

$$
\begin{aligned}
& \left|\int_{0}^{t} \int_{0}^{1}\left\{\left[\int_{0}^{x} v(y, \tau) d y\right]_{\tau} f(u)(\theta-\bar{\theta})\right\}(x, \tau) d x d \tau\right| \\
& \quad \leq \frac{1}{16} \int_{0}^{t} \int_{0}^{1}\left[\left(\int_{0}^{x} v(y, \tau) d y\right)_{\tau}\right]^{2}(x, \tau) d x d \tau+\Lambda \int_{0}^{t} \int_{0}^{1} \theta_{x}^{2}(x, \tau) d x d \tau \\
& \quad \leq \frac{1}{8} \int_{0}^{t} \int_{0}^{1}[f(u) \theta]^{2}(x, \tau) d x d \tau+\Lambda \int_{0}^{t} \int_{0}^{1}\left(v_{x}^{2}+\theta_{x}^{2}\right)(x, \tau) d x d \tau \\
& \quad \leq \frac{1}{8} \int_{0}^{t} \int_{0}^{1}[f(u) \theta]^{2}(x, \tau) d x d \tau+\Lambda, \quad t \in[0,+\infty) .
\end{aligned}
$$


Integrating by parts and using $(1.1)_{1},(2.4),(2.10),(2.16),(2.21),(2.22),(2.25)$, Hölder's inequality, and Cauchy's inequality, it follows that

$$
\begin{aligned}
\left|\int_{0}^{t} \int_{0}^{1}\left\{\left[\int_{0}^{x} v(y, \tau) d y\right]_{\tau} f(u) \bar{\theta}\right\}(x, \tau) d x d \tau\right| \\
\leq \Lambda+\Lambda \int_{0}^{t} \int_{0}^{1}\left[v_{x}^{2}+\int_{0}^{1} v^{2}(s, \tau) d s\right](x, \tau) d x d \tau \\
\quad+\frac{1}{C_{V}}\left|\int_{0}^{t} \int_{0}^{1}\left\{\left[\int_{0}^{x} v(y, \tau) d y\right] f(u)\left[\int_{0}^{1} \frac{1}{2} v^{2}(s, \tau) d s\right]_{\tau}\right\}(x, \tau) d x d \tau\right| \\
\leq \Lambda+\frac{1}{C_{V}} \mid \int_{0}^{t} \int_{0}^{1}\left\{\left[\left(\int_{0}^{x} v(y, \tau) d y\right)_{\tau} f(u)\right.\right. \\
\leq \Lambda+\frac{1}{16} \int_{0}^{t} \int_{0}^{1}\left\{\left[\int_{0}^{x} v(y, \tau) d y\right]_{\tau}\right\}(x, \tau) d x d \tau+\Lambda \int_{0}^{t} \int_{0}^{1}\left[v_{x}^{2}+v^{4}\right](x, \tau) d x d \tau \\
\leq \Lambda+\frac{1}{8} \int_{0}^{t} \int_{0}^{1}[f(u) \theta]^{2}(x, \tau) d x d \tau .
\end{aligned}
$$

(2.26)-(2.29) then imply (2.24).

LEMMA 2.5.

$$
\int_{0}^{1} v^{2}(x, t) d x \rightarrow 0 \quad \text { as } t \rightarrow+\infty .
$$

Proof. It is clear from (2.10) that

$$
\int_{0}^{+\infty} \int_{0}^{1} v^{2}(x, t) d x d t \leq \Lambda .
$$

Namely,

$$
\int_{0}^{1} v^{2}(x, t) d x \in L^{1}([0,+\infty)) .
$$

Multiplying $(1.1)_{2}$ by $v$ and integrating over [0,1], we obtain, with the help of (1.6) and (1.3),

$$
\begin{aligned}
& \left|\int_{0}^{1}\left(v v_{t}\right)(x, t) d x\right| \\
& \quad=\left|\int\left[\left(f(u) \theta-\hat{\mu}(u) v_{x}\right) v_{x}\right](x, t) d x\right| \\
& \quad \leq \Lambda \int_{0}^{1}\left[(f(u) \theta)^{2}+v_{x}^{2}\right](x, t) d x
\end{aligned}
$$

which, combined with (2.16) and (2.24), implies

$$
\int_{0}^{+\infty}\left|\frac{d}{d t} \int_{0}^{1} v^{2}(x, t) d x\right| d x \leq \Lambda .
$$


(2.31) and (2.33) yield (2.30).

LEMMA 2.6 .

$$
\begin{gathered}
\bar{\theta}(t)=\int_{0}^{1} \theta(x, t) d x \rightarrow \frac{E_{1}}{C_{V}}, \quad \text { as } t \rightarrow+\infty, \\
\int_{0}^{t} \int_{0}^{1}[f(u)]^{2}(x, \tau) d x d \tau \leq \Lambda, \quad t \in[0,+\infty), \\
\|f(u)(\cdot, t)\|_{L^{2}[0,1]} \rightarrow 0, \quad \text { as } t \rightarrow+\infty, \\
\|\hat{p}(u, \theta)(\cdot, t)\|_{L^{1}[0,1]}=\|(f(u) \theta)(\cdot, t)\|_{L^{1}[0,1]} \rightarrow 0, \quad \text { as } t \rightarrow+\infty .
\end{gathered}
$$

Proof. (2.34) follows from (2.22) and (2.30) directly. It is known from (2.34) that there exists $T_{0}>0$ such that

$$
\bar{\theta}(t) \geq \frac{E_{1}}{2 C_{V}} \quad \text { as } t \geq T_{0},
$$

which, together with (2.1), (2.11), (2.19), and (2.24), implies

$$
\begin{aligned}
\int_{T_{0}}^{t} & \int_{0}^{1}[f(u)]^{2}(x, \tau) d x d \tau \\
& \leq \frac{4 C_{V}^{2}}{E_{1}^{2}} \int_{T_{0}}^{t} \int_{0}^{1}[f(u) \bar{\theta}]^{2}(x, \tau) d x d \tau \\
& \leq \Lambda \int_{T_{0}}^{t} \int_{0}^{1}[f(u) \theta]^{2}(x, \tau) d x d \tau+\Lambda \int_{T_{0}}^{t} \int_{0}^{1}[f(u)(\theta-\bar{\theta})]^{2}(x, \tau) d x d \tau \\
& \leq \Lambda .
\end{aligned}
$$

(2.38) and (2.1) now yield (2.35).

To prove $(2.36)$, we make the following estimate by using $(1.1)_{1},(2.1),(2.16)$, and (2.35):

$$
\begin{aligned}
\int_{0}^{+\infty} & \left|\frac{d}{d t} \int_{0}^{1}[f(u)]^{2}(x, t) d x\right| d t \\
& \leq \Lambda+\Lambda \int_{0}^{+\infty} \int_{0}^{1} v_{x}^{2}(x, t) d x d t \leq \Lambda .
\end{aligned}
$$

(2.39) and (2.35) imply (2.36) directly.

$$
\|(f(u) \theta)(\cdot, t)\|_{L^{1}[0,1]} \leq\left(\int_{0}^{1}[f(u)]^{2}(x, t) d x\right)^{1 / 2} \cdot\left(\int_{0}^{1} \theta^{2}(x, t) d x\right)^{1 / 2} .
$$

This, combined with (2.11) and (2.36), gives (2.37).

So far, part I of Theorem 1.1 has been established by the above lemmas.

Next we will employ an idea of Andrews and Ball (see [AB]) and the results obtained above to prove part II of Theorem 1.1.

Suppose $\Psi \in L^{2}[0,1]$ with $\Psi \geq 0$ and $\Phi \in C^{2}([\underline{u}, \bar{U}])$ satisfying

$$
\Phi^{\prime}(z) f(z) \geq 0 \quad \text { for } z \in[\underline{u}, \bar{U}]
$$

where $\underline{u}$ and $\bar{U}$ are the lower and upper bounds of $u$. 
Let

$$
\varphi(x, t):=\int_{0}^{x} \Psi(y) \frac{\Phi^{\prime}(u(y, t))}{\hat{\mu}(u(y, t))} d y \quad \text { for } x \in[0,1], t \geq 0 .
$$

Multiplying $(1.2)_{2}$ with $\varphi$ and integrating over $[0,1] \times[0, t]$ and using the boundary condition (1.3), we get, with the help of (1.1) and integration by parts,

$$
\begin{aligned}
\int_{0}^{t} \int_{0}^{1} & {\left[(f(u) \theta) \Psi(x) \frac{\Phi^{\prime}(u)}{\hat{\mu}(u)}\right](x, \tau) d x d \tau } \\
= & \int_{0}^{1} v(x, t)\left[\int_{0}^{x} \Psi(y) \frac{\Phi^{\prime}(u(y, t))}{\hat{\mu}(u(y, t))} d y\right] d x-\int_{0}^{1} v_{0}(x)\left[\int_{0}^{x} \Psi(y) \frac{\Phi^{\prime}\left(u_{0}(y)\right)}{\hat{\mu}\left(u_{0}(y)\right)} d y\right] d x \\
& -\int_{0}^{t} \int_{0}^{1} v(x, \tau)\left[\int_{0}^{x} \Psi(y)\left(\frac{\Phi^{\prime}(u)}{\hat{\mu}(u)}\right)^{\prime}(y, \tau) v_{x}(y, \tau) d y\right] d x d \tau \\
& +\int_{0}^{1} \Psi(x) \Phi(u(x, t)) d x-\int_{0}^{1} \Psi(x) \Phi\left(u_{0}(x)\right) d x
\end{aligned}
$$

where' denotes the differentiation with respect to $u$.

To show the existence of the limit of the left-hand side of $(2.41)$ as $t \rightarrow+\infty$, we estimate each term on the right-hand side of (2.41).

For the first term, it is easy to see that

$$
\begin{aligned}
& \left|\int_{0}^{1} v(x, t)\left[\int_{0}^{x} \Psi(y) \frac{\Phi^{\prime}(u(y, t))}{\hat{\mu}(u(y, t))} d y\right] d x\right| \\
& \quad \leq\|v(\cdot, t)\|_{L^{2}[0,1]} \cdot\|\Psi\|_{L^{2}[0,1]} \cdot\left\|\frac{\Phi^{\prime}(u(\cdot, t))}{\hat{\mu}(u(\cdot, t))}\right\|_{L^{2}[0,1]} \\
& \quad \leq \Lambda\|v(\cdot, t)\|_{L^{2}[0,1]}
\end{aligned}
$$

which tends to zero as $t \rightarrow+\infty$, due to (2.30).

The third term can be treated as follows:

$$
\begin{aligned}
& \left|\int_{0}^{1} v(x, t)\left[\int_{0}^{x} \Psi(y)\left[\frac{\Phi^{\prime}(u)}{\hat{\mu}(u)}\right]^{\prime}(y, t) v_{x}(y, t) d y\right] d x\right| \\
& \leq\|v(\cdot, t)\|_{L^{2}}\|\Psi\|_{L^{2}} \cdot\left\|\left[\frac{\Phi^{\prime}(u)}{\hat{\mu}(u)}\right]^{\prime}(\cdot, t)\right\|_{L^{\infty}} \cdot\left\|v_{x}(\cdot, t)\right\|_{L^{2}} \\
& \leq \Lambda\left[\|v(\cdot, t)\|_{L^{2}}^{2}+\left\|v_{x}(\cdot, t)\right\|_{L^{2}}^{2}\right] \text { for all } t \geq 0 .
\end{aligned}
$$

Therefore, the limit of the third term as $t \rightarrow+\infty$ exists by $(2.10),(2.16)$, and the dominated convergence theorem.

It is obvious that the term $\mu_{0} \int_{0}^{1} \Psi(x) \Phi(u(x, t)) d x$ is uniformly bounded in $t \geq 0$ since $\underline{u} \leq u(x, t) \leq \bar{U}$.

Thus, the above estimates imply that

$$
\int_{0}^{t} \int_{0}^{1}\left\{[f(u) \theta] \Psi(x) \frac{\Phi^{\prime}(u)}{\hat{\mu}(u)}\right\}(x, \tau) d x d \tau \text { is bounded uniformly in } t \geq 0 .
$$


This, together with $(2.40),(2.2)$, and $\theta>0$, yields the existence of

$$
\lim _{t \rightarrow \infty} \int_{0}^{t} \int_{0}^{1}\left[f(u) \theta \Psi(x) \Phi^{\prime}(u)\right](x, \tau) d x d \tau .
$$

Furthermore, the existence of

$$
\lim _{t \rightarrow+\infty} \int_{0}^{1} \Psi(x) \Phi(u(x, t)) d x
$$

for all $\Psi \in C^{2}[0,1]$ with $\Psi \geq 0$, is established since each term in (2.41), apart from $\int_{0}^{1} \Psi(x) \Phi(u(x, t)) d x$, is either independent of $t$ or tends to a limit as $t \rightarrow+\infty$.

Therefore, it follows that

$$
\Phi(u(\cdot, t)) \rightarrow g_{\Phi}(\cdot) \quad \text { in } L^{2}[0,1]
$$

as $t \rightarrow+\infty$ for some $g_{\Phi} \in L^{2}[0,1]$.

In view of $\|\Phi(u(\cdot, t))\|_{L^{\infty}} \leq \Lambda$, it can be shown that

$$
g_{\Phi} \in L^{\infty}[0,1]
$$

and

$$
\Phi(u(\cdot, t)) \stackrel{*}{\rightarrow} g_{\Phi}(\cdot) \text { in } L^{\infty}[0,1]
$$

Let $\Phi \in C([\underline{u}, \bar{U}])$ be arbitrary and $\Psi \in L^{1}(0,1)$ now. It is easy to verify that

$$
\lim _{t \rightarrow \infty} \int_{0}^{1} \Psi(x) \Phi(u(x, t)) d x
$$

exists for all $\Psi \in L^{1}[0,1]$ and $\Phi \in C([\underline{u}, \bar{u}])$, by using the same method in $[\mathrm{AB}]$ and the following Lemma 2.7 which can be proved by the same argument as used for Lemma 3.1 in $[\mathrm{AB}]$.

Lemma 2.7. Let $f \in C(\mathbb{R})$ and let $0<\underline{u}<\bar{U}$. Then the set

$$
S=\operatorname{span}\left\{\Phi \in C^{2}([\underline{u}, \bar{U}]): \Phi^{\prime}(z) f(z) \geq 0 \text { if } z \in[\underline{u}, \bar{U}]\right\}
$$

is dense in $C([\underline{u}, \bar{U}])$.

Thus, it turns out that (2.42) holds for an arbitrary $\Phi \in C([\underline{u}, \bar{U}])$. The existence of probability measures $\nu_{x}$ follows at once from (2.42) and Theorem 5 in Tartar's paper in 1979 ([TA]). To prove that $\operatorname{supp} \nu_{x} \subset K=\{z: f(z)=0\}$ a.e., it suffices to show that if $\Phi$ is zero on $K$ then $\left\langle\nu_{x}, \Phi\right\rangle=0$ a.e. But, if $\Phi$ is zero on $K$, then $\Phi(u(\cdot, t)) \rightarrow 0$ in measure as $t \rightarrow+\infty$ due to (2.36). Therefore, $\Phi(u(\cdot, t)) \rightarrow 0$ in $L^{\infty}[0,1]$ as $t \rightarrow \infty$, and hence $\left\langle\nu_{x}, \Phi\right\rangle=0$, a.e., as required.

Theorem 1.1 has been proved completely now.

Corollaries 1.3 and 1.4 can be proved in the same way as in $[\mathrm{AB}]$. 
3. The proof of Theorem 1.5. In view of (1.10), (2.1), and the smoothness of $f^{\prime}(u)$, there exists a constant $b>0$ such that

$$
-f^{\prime}(u) \geq b>0 \text { for } u \in[\underline{u}, \bar{U}] .
$$

LEMMA 3.1. If (1.10) holds, then

$$
\int_{0}^{1} u_{x}^{2}(x, t) d x+\int_{0}^{t} \int_{0}^{1}\left(u_{x}^{2}+\theta u_{x}^{2}\right)(x, \tau) d x d \tau \leq \Lambda, \quad t \in[0,+\infty) .
$$

Proof. Define $M(u)=\int_{\underline{u}}^{u} \hat{\mu}(\xi) d \xi$ and consider $M(u)$ as a function of $x$ and $t$. Then we may rewrite $(1.1)_{2}$ as

$$
\left[v-M(u)_{x}\right]_{t}=[-f(u) \theta]_{x} .
$$

Multiply the above equation by $\left[v-(M u)_{x}\right]$ and then integrate over $[0,1] \times[0, t]$. We arrive at

$$
\begin{aligned}
\frac{1}{2} \int_{0}^{1}\left[v-M(u)_{x}\right]^{2}(x, t) d x+\int_{0}^{t} \int_{0}^{1}\left\{\left[-f^{\prime}(u) \theta \hat{\mu}(u)\right] u_{x}^{2}\right\}(x, \tau) d x d \tau \\
=\frac{1}{2} \int_{0}^{1}\left[v-(M u)_{x}\right]^{2}(x, 0) d x-\int_{0}^{t} \int_{0}^{1}\left[f^{\prime}(u) \theta u_{x} v\right](x, \tau) d x d \tau \\
\quad-\int_{0}^{t} \int_{0}^{1}\left[f(u) v \theta_{x}\right](x, \tau) d x d \tau+\int_{0}^{t} \int_{0}^{1}\left[f(u) \hat{\mu}(u) u_{x} \theta_{x}\right](x, \tau) d x d \tau
\end{aligned}
$$

(2.2), (2.5), (2.10), (2.11), and (3.1) then yield

$$
\begin{gathered}
\frac{1}{2} \int_{0}^{1}\left[v-M(u)_{x}\right]^{2}(x, t) d x+\frac{b \mu_{1}}{2} \int_{0}^{t} \int_{0}^{1}\left[\theta u_{x}^{2}\right](x, \tau) d x d \tau \\
\leq \Lambda+\Lambda \int_{0}^{t} \int_{0}^{1} \frac{\theta_{x}^{2}}{\theta}(x, \tau) d x d \tau \\
\leq \Lambda+\Lambda \int_{0}^{t} \int_{0}^{1}\left[\theta_{x}^{2}+\frac{\theta_{x}^{2}}{\theta^{2}}\right](x, \tau) d x d \tau \leq \Lambda
\end{gathered}
$$

which, with the help of (2.1) and (2.4), implies

$$
\int_{0}^{1} u_{x}^{2}(x, t) d x+\int_{0}^{t} \int_{0}^{1} \theta u_{x}^{2}(x, \tau) d x d \tau \leq \Lambda, \quad t \in[0,+\infty) .
$$

Next, it is known from (2.34) that there exists a $T_{0}>0$ such that

$$
\bar{\theta}(t)=\int_{0}^{1} \theta(x, t) d x=\theta(z(t), t) \geq \frac{E_{1}}{2 C_{V}}>0 \quad \text { for } t \geq T_{0} .
$$

Then, it can be shown by the Hölder inequality that

$$
\begin{aligned}
\theta^{1 / 2}(x, t) & =\theta^{1 / 2}(z(t), t)+\int_{z(t)}^{x} \frac{\theta_{x}(\xi, t)}{2 \theta^{1 / 2}(\xi, t)} d \xi \\
& \geq\left(\frac{E_{1}}{2 C_{V}}\right)^{1 / 2}\left[1-\frac{1}{2}\left(\int_{0}^{1} \frac{\theta_{x}^{2}}{\theta^{2}}(x, t) d x\right)^{1 / 2}\right]
\end{aligned}
$$


which implies

$$
\theta^{1 / 2}(x, t)+\left[\int_{0}^{1} \frac{\theta_{x}^{2}}{\theta^{2}}(x, t) d x\right]^{1 / 2} \geq \Lambda>0, \quad \text { for } t \geq T_{0} .
$$

By using the Cauchy inequality, it then follows that

$$
\theta(x, t)+\int_{0}^{1} \frac{\theta_{x}^{2}}{\theta^{2}}(x, t) d x \geq \Lambda>0, \quad t \in\left[\tau_{0},+\infty\right) .
$$

Therefore,

$$
u_{x}^{2}(x, t) \leq \Lambda\left\{\theta u_{x}^{2}(x, t)+u_{x}^{2}(x, t) \int_{0}^{1} \frac{\theta_{x}^{2}}{\theta^{2}}(x, t) d x\right\}, \quad t \in\left[T_{0},+\infty\right) .
$$

This, combined with (2.5) and (3.5), implies

$$
\int_{T_{0}}^{t} \int_{0}^{1} u_{x}^{2}(x, \tau) d x d \tau \leq \Lambda, \quad t \in\left[\tau_{0},+\infty\right) .
$$

Thus, (3.2) follows from (3.5) and (3.7).

LEMMA 3.2. If (1.10) holds, then there exists a $T^{*}>0$ such that

$$
\int_{0}^{1}\left(v_{x}^{2}+\theta_{x}^{2}\right)(x, t) d x+\int_{T^{*}}^{t} \int_{0}^{1}\left[v_{x x}^{2}+\theta_{t}^{2}\right](x, \tau) d x d \tau \leq \Lambda \quad \text { for } t \geq T^{*}
$$

and

$$
\lim _{t \rightarrow+\infty} \int_{0}^{1}\left(v_{x}^{2}+\theta_{x}^{2}\right)(x, t) d x \rightarrow 0 \quad \text { as } t \rightarrow+\infty .
$$

Proof. Multiplying $(1.1)_{2}$ by $\left(-f(u) \theta+\hat{\mu}(u) v_{x}\right)_{x}$ and using $(1.1)_{1},(1.3),(2.1),(2.2)$, and Cauchy's inequality, we get

$$
\begin{aligned}
\int_{T}^{t} \int_{0}^{1}\left[\hat{\mu}(u) v_{x x}\right]^{2}(x, \tau) d x d \tau+\frac{1}{2} \int_{0}^{1}\left(\hat{\mu}(u) v_{x}^{2}\right)(x, t) d x \\
\leq \frac{1}{2} \int_{0}^{1}\left[\hat{\mu}(u) v_{x}^{2}\right](x, T) d x+\int_{T}^{t} \int_{0}^{1}\left[\frac{1}{2} \hat{\mu}^{\prime}(u) v_{x}^{3}\right](x, \tau) d x d \tau \\
\quad+\int_{T}^{t} \int_{0}^{1}\left[f(u) \theta v_{x t}\right](x, \tau) d x d \tau+\frac{\mu_{1}^{2}}{4} \int_{T}^{t} \int_{0}^{1} v_{x x}^{2}(x, \tau) d x d \tau \\
+\Lambda \int_{T}^{t}\left[\max _{[0,1]} v_{x}^{2}(\cdot, \tau) \int_{0}^{1} u_{x}^{2}(x, \tau) d x\right] d \tau+\Lambda \int_{T}^{t} \int_{0}^{1} \theta_{x}^{2}(x, \tau) d x d \tau \\
+\Lambda \int_{T}^{t}\left[\max _{[0,1]} \theta^{2}(\cdot, \tau) \int_{0}^{1} u_{x}^{2}(x, \tau) d x\right] d \tau \quad \text { for any } t \geq T>0 .
\end{aligned}
$$

To estimate the terms in (3.10), we first give an estimate on $\int_{0}^{t} \int_{0}^{1} v_{x}^{4}(x, \tau) d x d \tau$, which plays a key role in the following estimates. Due to $W^{1,1} \hookrightarrow L^{\infty}$, it follows that

$$
\begin{aligned}
& \int_{T}^{t} \int_{0}^{1} v_{x}^{4}(x, \tau) d x d \tau \leq \int_{T}^{t} \max _{[0,1]} v_{x}^{2}\left(\int_{0}^{1} v_{x}^{2} d x\right) d \tau \\
& \quad \leq \Lambda \sup _{\tau \in[T, t]} \int_{0}^{1} v_{x}^{2}(x, \tau) d x \cdot\left[\int_{T}^{t} \int_{0}^{1}\left[v_{x}^{2}+v_{x x}^{2}\right]\right](x, \tau) d x d \tau \quad \text { for any } t \geq T>0 .
\end{aligned}
$$


Similarly, it can be shown, with the help of $W^{1,1} \hookrightarrow L^{\infty}$ and Cauchy's inequality, that

$$
\begin{aligned}
& \int_{T}^{t} \max _{[0,1]} v_{x}^{2}(\cdot, \tau) d \tau \\
& \quad \leq \Lambda(\delta) \int_{T}^{t} \int_{0}^{1} v_{x}^{2} d x d \tau+\delta \int_{T}^{t} \int_{0}^{1} v_{x x}^{2} d x d \tau \\
& \quad \text { for any } t \geq T>0 \text { and } \delta>0
\end{aligned}
$$

It reads from $(2.19),(2.22)$, and $(2.11)$ that

$$
\max _{[0,1]} \theta(\cdot, t) \leq \Lambda+\left(\int_{0}^{1} \theta_{x}^{2}(x, t) d x\right)^{1 / 2} \quad \text { for } t \geq 0
$$

Moreover, (2.4) and (3.2) yield

$$
\int_{0}^{1}\left[\theta^{2}(x, t)+u_{x}^{2}(x, t)\right] d x \leq \Lambda \quad \text { for } t \geq 0 .
$$

We turn to estimate the terms in (3.10) by using these inequalities (3.11)-(3.14) in which $\delta$ can be chosen suitably.

Using $(1.1)_{1},(2.1),(2.4),(3.12),(3.13)$, Cauchy's inequality, and integration by parts, we obtain

$$
\begin{aligned}
\int_{T}^{t} \int_{0}^{1}[ & \left.f(u) \theta v_{x t}\right](x, \tau) d x d \tau \\
= & \int_{0}^{1}\left[f(u) \theta v_{x}\right](x, t) d x-\int_{0}^{1}\left[f(u) \theta v_{x}\right](x, T) d x \\
& \quad-\int_{T}^{t} \int_{0}^{1} v_{x}\left[f^{\prime}(u) \theta v_{x}+f(u) \theta_{t}\right] d x d \tau \\
\leq & \frac{\mu_{1}}{4} \int_{0}^{1} v_{x}^{2}(x, t) d x+\Lambda \max \theta(x, t) \int_{0,1]}^{1}|f(u) \theta|(x, t) d x \\
& +\Lambda \int_{0}^{1}\left\{[f(u) \theta]^{2}+v_{x}^{2}\right\}(x, T) d x+\Lambda \int_{T}^{t} \max v_{x}^{2}\left(\int_{0}^{1} \theta d x\right) d \tau \\
& +\Lambda \int_{T}^{t} \int_{0}^{1} v_{x}^{2} d x d \tau+\frac{C_{V}}{4} \int_{T}^{t} \int_{0}^{1} \theta_{t}^{2} d x d \tau \\
\leq & \frac{\mu_{1}}{4} \int_{0}^{1} v_{x}^{2}(x, t) d x+\Lambda \int_{0}^{1}\left[(f(u) \theta)^{2}+v_{x}^{2}\right](x, T) d x+\Lambda \int_{0}^{1}|f(u) \theta|(x, t) d x \\
& \quad+\frac{k}{4} \int_{0}^{1} \theta_{x}^{2}(x, t) d x+\Lambda\left[\int_{0}^{1}|f(u) \theta|(x, t) d x\right]^{2}+\Lambda \int_{T}^{t} \int_{0}^{1} v_{x}^{2} d x d \tau \\
& +\frac{\mu_{1}^{2}}{8} \int_{T}^{t} \int_{0}^{1} v_{x x}^{2} d x d \tau+\frac{C_{V}}{4} \int_{T}^{t} \int_{0}^{1} \theta_{t}^{2} d x d \tau \text { for any } t \geq T>0,
\end{aligned}
$$

whereafter, $k$ is the constant in (1.6). 
By using (2.1), (3.11), and Cauchy's inequality, we get

$$
\begin{aligned}
& \left|\int_{T}^{t} \int_{0}^{1} \frac{1}{2} \hat{\mu}^{\prime}(u) v_{x}^{3} d x d \tau\right| \\
& \quad \leq \Lambda \int_{T}^{t} \int_{0}^{1} v_{x}^{2} d x d \tau+\Lambda \int_{T}^{t} \int_{0}^{1} v_{x}^{4} d x d \tau \\
& \quad \leq \Lambda \int_{T}^{t} \int_{0}^{1} v_{x}^{2} d x d \tau+\Lambda \sup _{\tau \in[T, t]} \int_{0}^{1} v_{x}^{2}(x, \tau) d x \cdot\left[\int_{T}^{t} \int_{0}^{1}\left(v_{x}^{2}+v_{x x}^{2}\right) d x d \tau\right] \\
& \quad \text { for } t \geq T>0
\end{aligned}
$$

Similarly, the fifth and seventh terms on the right-hand side of (3.10) can be bounded by

$$
\Lambda \int_{T}^{t} \int_{0}^{1} v_{x}^{2} d x d \tau+\frac{\mu_{1}^{2}}{8} \int_{T}^{t} \int_{0}^{1} v_{x x}^{2} d x d \tau
$$

and

$$
\Lambda \int_{T}^{t} \int_{0}^{1}\left(u_{x}^{2}+\theta_{x}^{2}\right) d x d \tau
$$

by using (3.12) and (3.14), and (3.13)-(3.14), respectively.

This, together with (3.10)-(3.16) and Cauchy's inequality, implies

$$
\begin{aligned}
& \frac{\mu_{1}^{2}}{2} \int_{T}^{t} \int_{0}^{1} v_{x x}^{2} d x d \tau+\frac{\mu_{1}}{4} \int_{0}^{1} v_{x}^{2}(x, t) d x \\
& \leq \Lambda \int_{0}^{1}\left[(f(u) \theta)^{2}+v_{x}^{2}\right](x, T) d x+\Lambda\left[\int_{0}^{1}|f(u) \theta|(x, t) d x+\left(\int_{0}^{1}|f(u) \theta|(x, t) d x\right)^{2}\right] \\
& \quad+\Lambda \int_{T}^{t} \int_{0}^{1}\left[u_{x}^{2}+v_{x}^{2}+\theta_{x}^{2}\right](x, \tau) d x d \tau \\
& \quad+\Lambda \sup _{\tau \in[T, t]} \int_{0}^{1} v_{x}^{2}(x, \tau) d x \cdot\left[\int_{T}^{t} \int_{0}^{1}\left(v_{x}^{2}+v_{x x}^{2}\right) d x d \tau\right] \\
& \quad+\frac{k}{4} \int_{0}^{1} \theta_{x}^{2}(x, t) d x+\frac{C_{V}}{4} \int_{T}^{t} \int_{0}^{1} \theta_{t}^{2}(x, \tau) d x d \tau .
\end{aligned}
$$

Multiplying $(2.7)$ by $\theta_{t}$ and integrating over $[0,1] \times[T, t]$, we get, with the help of (1.3), (3.11), (3.12), (3.14), and the Cauchy inequality, 


$$
\begin{aligned}
& \frac{k}{2} \int_{0}^{1} \theta_{x}^{2}(x, t) d x+\frac{C_{V}}{2} \int_{T}^{t} \int_{0}^{1} \theta_{t}^{2}(x, \tau) d x d \tau \\
& \leq \frac{k}{2} \int_{0}^{1} \theta_{x}^{2}(x, T) d x+\Lambda \int_{T}^{t} \max _{[0,1]} v_{x}^{2}(\cdot, \tau)\left(\int_{0}^{1} \theta^{2}(x, \tau) d x\right) d \tau \\
& \quad+\Lambda \int_{T}^{t} \int_{0}^{1} v_{x}^{4}(x, \tau) d x d \tau \\
& \leq \frac{k}{2} \int_{0}^{1} \theta_{x}^{2}(x, T) d x+\frac{\mu_{1}^{2}}{4} \int_{T}^{t} \int_{0}^{1} v_{x x}^{2}(x, \tau) d x d \tau+\Lambda \int_{T}^{t} \int_{0}^{1} v_{x}^{2}(x, \tau) d x d \tau \\
& \quad+\Lambda \sup _{\tau \in[T, t]} \int_{0}^{1} v_{x}^{2}(x, \tau) d x\left[\int_{T}^{t} \int_{0}^{1}\left(v_{x}^{2}+v_{x x}^{2}\right)(x, \tau) d x d \tau\right] \text { for any } t \geq T>0
\end{aligned}
$$

(3.17) and (3.18) imply

$$
\begin{aligned}
& \int_{0}^{1}\left(v_{x}^{2}+\right.\left.\theta_{x}^{2}\right)(x, t) d x+\int_{T}^{t} \int_{0}^{1}\left(v_{x x}^{2}+\theta_{t}^{2}\right)(x, \tau) d x d \tau \\
& \leq \Lambda \int_{0}^{1}\left[v_{x}^{2}+\theta_{x}^{2}+(f(u) \theta)^{2}\right](x, T) d x+\int_{0}^{1}|f(u) \theta|(x, t) d x+\left(\int_{0}^{1}|f(u) \theta|(x, t) d x\right)^{2} \\
&+\int_{T}^{t} \int_{0}^{1}\left(u_{x}^{2}+v_{x}^{2}+\theta_{x}^{2}\right)(x, \tau) d x d \tau \\
&+\sup _{\tau \in[T, t]} \int_{0}^{1} v_{x}^{2}(x, \tau) d x \cdot\left[\int_{T}^{t} \int_{0}^{1}\left(v_{x}^{2}+v_{x x}^{2}\right)(x, \tau) d x d \tau\right] \\
& \text { for any } t \geq T>0 .
\end{aligned}
$$

Due to Lemma 3.1 and the results in Sec. 2, it is known that

$$
\int_{0}^{+\infty} \int_{0}^{1}\left\{[f(u) \theta]^{2}+u_{x}^{2}+v_{x}^{2}+\theta_{x}^{2}\right\} d x d \tau \leq \Lambda
$$

and

$$
\int_{0}^{1}|f(u) \theta|(x, t) d x \rightarrow 0 \quad \text { as } t \rightarrow+\infty .
$$

Therefore, for any $\varepsilon>0$, there exists a $T_{1}>0$ such that the following holds:

$$
\begin{gathered}
\Lambda \int_{0}^{1}\left\{[f(u) \theta]^{2}+v_{x}^{2}+\theta_{x}^{2}\right\}\left(x, T_{1}\right) d x<\varepsilon \\
\Lambda\left\{\int_{0}^{1}|f(u) \theta|(x, t) d x+\left[\int_{0}^{1}|f(u) \theta|(x, t) d x\right]^{2}\right\}<\varepsilon \quad \text { for any } t \geq T_{1}, \\
\Lambda \int_{T}^{t} \int_{0}^{1}\left[u_{x}^{2}+v_{x}^{2}+\theta_{x}^{2}\right] d x d \tau<\varepsilon \quad \text { for any } t \geq T_{1} .
\end{gathered}
$$

For convenience, we assume that the constant $\Lambda$ in (3.19)-(3.22) satisfies $\Lambda \geq 1$. 
We choose $\varepsilon$ so small that

$$
\varepsilon<\frac{1}{10}
$$

It follows from (3.20) that

$$
\int_{0}^{1}\left(v_{x}^{2}+\theta_{x}^{2}\right)\left(x, T_{1}\right) d x<\frac{\varepsilon}{\Lambda} \leq \varepsilon
$$

Let us define

$$
T_{2}=\sup \left\{t: \sup _{\tau \in\left[T_{1}, t\right]} \int_{0}^{1}\left(v_{x}^{2}+\theta_{x}^{2}\right)(x, \tau) d x \leq 5 \varepsilon\right\}
$$

and show next that $T_{2}=+\infty$.

Suppose that $T_{2}<+\infty$. By taking $t=T_{2}$ and $T=T_{1}$ in (3.19) and using (3.20)(3.23), it turns out that

$$
\begin{aligned}
& \int_{0}^{1}\left(\theta_{x}^{2}+v_{x}^{2}\right)\left(x, T_{2}\right) d x+\frac{1}{2} \int_{T_{1}}^{T_{2}} \int_{0}^{1} v_{x x}^{2} d x d \tau+\int_{T_{1}}^{T_{2}} \int_{0}^{1} \theta_{t}^{2} d x d \tau \\
& \leq 3 \varepsilon+5 \varepsilon^{2}<3 \varepsilon+\frac{\varepsilon}{2}<4 \varepsilon .
\end{aligned}
$$

Namely, $\int_{0}^{1}\left(\theta_{x}^{2}+v_{x}^{2}\right)\left(x, T_{2}\right) d x<4 \varepsilon$, which contradicts the definition of $T_{2}$. Then $T_{2}=$ $+\infty$.

This implies that

$$
\int_{0}^{1}\left(\theta_{x}^{2}+v_{x}^{2}\right)(x, t) d x \leq 5 \varepsilon \quad \text { for } t \geq T_{1}
$$

which yields, due to the arbitrary smallness of $\varepsilon$, that

$$
\int_{0}^{1}\left(\theta_{x}^{2}+v_{x}^{2}\right)(x, t) d x \rightarrow 0 \quad \text { as } t \rightarrow+\infty .
$$

(3.8) can be obtained from (3.19), (3.25) and the above arguments. The proof of Lemma 3.2 is finished then.

We prove Theorem 1.5 now. It has been proved that

$$
\|v(\cdot, t)\|_{H^{1}[0,1]} \rightarrow 0 \quad \text { as } t \rightarrow+\infty .
$$

It is known from part I of Theorem 1.1 that

$$
\int_{0}^{1}[f(u)]^{2}(x, t) d x \rightarrow 0 \text { as } t \rightarrow+\infty .
$$

On the other hand, due to (3.1) and the mean value theorem, it can be shown that

$$
\int_{0}^{1}[f(u)]^{2}(x, t) d x \geq b^{2} \int_{0}^{1}(u-\hat{u})^{2}(x, t) d x
$$

where $\hat{u}$ is the unique root of $f(u)=0$ in $[\tilde{u}, \widetilde{U}]$. Thus,

$$
\int_{0}^{1}(u-\hat{u})^{2}(x, t) d x \rightarrow 0 \quad \text { as } t \rightarrow+\infty .
$$


Furthermore, it follows from Lemma 3.1 that $\int_{0}^{+\infty} \int_{0}^{1} u_{x}^{2} d x d \tau \leq \Lambda<+\infty$, while Lemma 3.2 and $(1.1)_{1}$ imply

$$
\begin{aligned}
\int_{T^{*}}^{+\infty}\left|\frac{d}{d t} \int_{0}^{1} u_{x}^{2}(x, t) d x\right| d t & \leq \Lambda \int_{T^{*}}^{+\infty} \int_{0}^{1} u_{x}^{2} d x d t+\Lambda \int_{T^{*}}^{+\infty} \int_{0}^{1} v_{x x}^{2} d x d t \\
& \leq \Lambda<+\infty
\end{aligned}
$$

Therefore,

$$
\int_{0}^{1} u_{x}^{2}(x, t) d x \rightarrow 0 \quad \text { as } t \rightarrow+\infty
$$

(3.26) and (3.27) yield

$$
\|u(\cdot, t)-\hat{u}\|_{H^{1}}^{2} \rightarrow 0 .
$$

It is known from part I of Theorem 1.1 that

$$
\bar{\theta}(t)=\int_{0}^{1} \theta(x, t) d x \rightarrow \frac{E_{1}}{C_{V}} \quad \text { as } t \rightarrow+\infty .
$$

This, combined with (3.9), implies

$$
\left\|\theta(\cdot, t)-\frac{E_{1}}{c_{V}}\right\|_{L^{2}[0,1]} \leq\left[\int_{0}^{1} \theta_{x}^{2}(x, t) d x\right]^{1 / 2}+\left\|\bar{\theta}(t)-\frac{E}{C_{V}}\right\|_{L^{2}} \rightarrow 0 \text { as } t \rightarrow+\infty
$$

and furthermore,

$$
\left\|\theta(\cdot, t)-\frac{E_{1}}{c_{V}}\right\|_{H^{1}} \rightarrow 0 \quad \text { as } t \rightarrow+\infty
$$

So far, we have proved that all of $(u-\hat{u}), v$, and $\left(\theta-\frac{E_{1}}{C_{V}}\right)$ become small in the $H^{1}$ norm for large $t$. Thus, the arguments similar to those in $[\mathrm{OK}]$ can be used to obtain the exponential convergence of $\{u, v, \theta\}$ to the constant state $\left\{\hat{u}, 0, \frac{E_{1}}{C_{V}}\right\}$ as $t \rightarrow+\infty$. We omit the details.

\section{REFERENCES}

[AB] G. Andrews and J. M. Ball, Asymptotic behavior and changes of phase in one-dimensional nonlinear viscoelasticity, J. Differential Equations 44, 306-341 (1982)

[DA] C. M. Dafermos, Global smooth solutions to the initial boundary value problem for the equations of one-dimensional nonlinear thermoviscoelasticity, SIAM J. Math. Anal. 13, 397-408 (1982)

[DH] C. M. Dafermos and L. Hsiao, Global smooth thermomechanical processes in one-dimensional nonlinear thermoviscoelasticity, Nonlinear Anal. T.M.A. 6, 435-454 (1982)

[GM] J. M. Greenberg and R. C. MacCamy, On the exponential stability of solutions of $E\left(u_{x}\right) u_{x x}+$ $\lambda u_{x t x}=\rho u_{t t}$, J. Math. Appl. 31, 406-417 (1970)

[NA] T. Nagasawa, On the one-dimensional motion of polytropic ideal gas non-fixed on the boundary, J. Differential Equations 65, No. 1, 49-67 (1986)

[OK] M. Okada and S. Kawashima, On the equation of one-dimensional motion of compressible viscous fluids, J. Math. Kyoto Univ. 23, 55-71 (1983)

[TA] L. Tartar, Compensated compactness and applications to partial differential equations, in Nonlinear Analysis and Mechanics: Heriot-Watt Symposium, Vol. IV, pp. 136-212, Research Notes in Math., Vol. 39, Pitman, Boston, London, 1979 